\title{
Occupational therapy for adults with intellectual disabilities and sensory processing challenges: a Delphi study exploring practice within acute assessment and treatment units
}

\author{
Helen Fustice \\ Learning Disability and Neurodevelopmental Service, Sussex Partnership NHS Foundation Trust, Worthing, UK \\ David Haines and fon Wright \\ School of Health Sciences, University of Brighton, Eastbourne, UK
}

\begin{abstract}
Purpose - Lack of research means guidance regarding the most effective sensory interventions for adults with intellectual disabilities is limited. Preliminary consensus evidence was created by gathering experienced occupational therapists' views on practice. The purpose of this study was to establish important elements of practice when using sensory integration-informed approaches on assessment and treatment units.

Design/methodology/approach - A modified Delphi process was used to gather, explore and synthesise the views of 13 occupational therapists. Data was collected via online surveys and included quantitative importance ratings and qualitative comments.

Findings - An experienced panel was in strong agreement regarding many elements of practice they felt were important for use in this setting, and a list of important practice items was created. Two themes were identified: "Complexity" and "Pragmatism vs. Ideal World." The themes highlight challenges to practice in this area and the complex clinical reasoning used to overcome these.

Research limitations/implications - Participants disagreed about the level of importance of a substantial number of items, therefore items that did not reach consensus may still be important.

Practical implications - This study indicates potentially helpful clinical tools, facilities and training and identifies support needed for occupational therapists working in these settings.

Originality/value - This study contributes to the body of knowledge regarding how occupational therapists do and could work with people with intellectual disabilities and sensory integration difficulties. Although this study takes a UK and Ireland perspective, similar agendas regarding care and support for those with intellectual disabilities internationally mean findings are relevant more widely.
\end{abstract}

Keywords Intellectual disabilities, Occupational therapy, Inpatient, Sensory processing, Assessment and treatment unit, Sensory integration, Learning disabilities, Delphi

Paper type Research paper

\section{Introduction}

People with intellectual disabilities have life-long and significant challenges with intellectual functioning and adaptive skills, starting before adulthood (Johnson et al., 2019). The quality of care for people with intellectual disabilities is a current priority in the UK and Ireland and internationally (Lakhan and Ekúndayò, 2017; NHS, 2019). Particular attention is paid to those with intellectual disabilities and a mental health condition and/or behaviour described as challenging. This group is at particular risk of admission to inpatient services and is a key focus for agendas aiming to ensure people can live successfully in the community. Within the UK and Ireland a variety of in-patient units exists, including

The current issue and full text archive of this journal is available on Emerald Insight at: https://www.emerald.com/insight/2398-8819.htm

Irish Journal of Occupational Therapy

49/1 (2021) 28-35

Emerald Publishing Limited [ISSN 2398-8819]

[DOI 10.1108/IJOT-11-2020-0018] forensic, acute, rehabilitation and continuing care. The study described in this paper focuses on specialist acute assessment and treatment units (ATUs), where aimed-for length of stay is typically 3-6 months (although is frequently much longer). Attempts to reduce the number of people with intellectual

(C) Helen Justice, David Haines and Jon Wright. Published in Irish fournal of Occupational Therapy. Published by Emerald Publishing Limited. This article is published under the Creative Commons Attribution (CC BY 4.0) licence. Anyone may reproduce, distribute, translate and create derivative works of this article (for both commercial and non-commercial purposes), subject to full attribution to the original publication and authors. The full terms of this licence maybe seen at http://creativecommons.org/licences/ by/4.0/legalcode

Helen Justice was funded by a National Institute for Health Research (NIHR) MRes for this research project - grant reference CATMASTERS-2012-05-107. This publication presents independent research funded by the National Institute for Health Research (NIHR). The views expressed are those of the authors and not necessarily those of the NHS, the NIHR or the Department of Health and Social Care.

Received 18 November 2020

Revised 25 February 2021

Accepted 11 March 2021 
disabilities within in-patient settings have been ineffective in many areas (NHS Digital, 2020). The paucity of occupational therapy research in intellectual disabilities is well documented (Haines et al., 2018). As professionals working in ATUs, it is vital to improve the evidence-base to support provision of best care in these settings.

Therapeutic strategies that improve quality of life and reduce restrictive practices are promoted as part of the international agenda to enhance care for this population (Bowen and Kemp, 2014). Some authors have suggested sensory approaches can support this (Gawlas and Blaskowitz, 2020; McGill and Breen, 2020); however, lack of research in this area means guidance is unavailable regarding the most effective sensory interventions for those with intellectual disabilities (NICE, 2015).

This study endeavoured to create preliminary evidence by gathering experienced occupational therapists' views on practice. The research sought to establish important elements of practice when using sensory integration-informed approaches with adults with intellectual disabilities admitted to ATUs.

Sensory approaches are diverse and involve varying types of intervention, based on different theoretical constructs. Some key approaches include Ayres Sensory Integration $\left(\mathrm{ASI}^{\circledR}\right.$ ) (also described as sensory integration therapy); sensory-based interventions; sensory modulation; sensory stimulation; and sensory activity. ASI ${ }^{\circledR}$ is based on Ayres's (1973) theories that some people have difficulties integrating sensory information from the world around them and their own bodies. Ayres postulated these difficulties can lead to problems with attention, emotional regulation, body image, posture, motorplanning and coordination. ASI ${ }^{\circledR}$ is a manualised, active, clientled, play-based intervention, aiming to provide a "just right" challenge to elicit an adaptive response from an individual in a sensory-rich clinic space (Parham et al., 2011). Sensory-based interventions may also be based on Ayres's theories, but use sensory modalities in the individual's own environment (Watling and Hauer, 2015), tend to be therapist or parent/ carer-led and do not require an adaptive response. Sensory modulation (Brown et al., 2018) is a term increasingly used within the mental health literature describing a variety of sensory interventions with the aim of reducing distress. Sensory stimulation meanwhile aims to provide an accessible, nondirective activity by stimulating the senses. It arose from the concept of Snoezelen and is predominantly used in multisensory environments (Breslin et al., 2020). Use of sensory activity is another approach, recognising that individuals with significant cognitive challenges may engage in activity at a sensory level of functioning (Haines, 2018), where the focus is on supporting the process of an activity and its sensory qualities rather than the end result.

Lack of consensus regarding language use when describing sensory approaches means many of the above terms have been used interchangeably within the literature. This has led to confusion about what particular sensory approaches do and do not involve and causes problems when assessing their effectiveness, e.g. debate regarding which articles should be included/excluded in systematic reviews (Watling and Hauer, 2015). For the purposes of this paper, the term sensory integration difficulties will be used to describe difficulties integrating sensory information. Sensory integration-informed approaches will be used to describe both $\mathrm{ASI}^{\circledR}$ and use of sensory-based interventions using clinical reasoning based on Ayres's theories, whilst sensory approaches will be used as an umbrella term to describe use of all sensory techniques described above.

\section{Literature review}

A broad and inclusive review of the sensory integration literature related to intellectual disability was conducted owing to a dearth of published material in this area. This included mental health and autism literature, as those admitted to ATUs frequently have these additional diagnoses. The CINAHL, AMED, PsychINFO, Medline, OT Seeker, Cochrane and Pubmed Electronic databases were searched using a comprehensive range of search terms and reference lists of articles were also hand-searched, uncovering 35 relevant articles.

Research points to some people with intellectual disabilities having sensory integration difficulties, though estimates of incidences vary. Reisman and Hanschu (1993) and Green et al. (2003) found $40 \%$ and $21 \%$ incidence of sensory integration difficulties, respectively, in samples within a long-term institution in the USA and on the caseloads of community intellectual disability occupational therapists in the UK. Although often using small samples, an increasing number of mental health studies have found differences in sensory-related indicators amongst those with a variety of mental health conditions (e.g. Brown et al., 2020). Meanwhile, studies involving healthy adults suggest difficulties with sensory integration may be related to mood, pain, impulsivity and impact on participation in daily activities and quality of life (e.g. Hebert, 2015). Prevalence of sensory differences in autism has been more widely researched and is estimated to be up to $96 \%$ (Watling and Hauer, 2015) and highly prevalent in lowfunctioning autistic adults (Gonthier, 2016).

Although participants in many of the above studies may not be directly representative of those admitted to ATUs, the high incidence of co-occurring mental health conditions and autism amongst inpatients with intellectual disabilities (NHS Digital, 2020) suggests the contribution of sensory integration difficulties to functional or behavioural challenges should be of interest to professionals working in this setting. This is supported by NICE (2015) guidance recommending consideration of individuals' sensory needs when working with those with intellectual disability and behaviour that challenges.

Little recent literature exists regarding sensory integration intervention with adults with intellectual disabilities. Within the UK Green et al. (2003) and Urwin and Ballinger (2005) reported on therapy in community settings; Soper and Thorley (1996) a long-stay hospital. A larger number of North American studies involved participants within large residential facilities (e.g. Reisman, 1993). Outcomes included reductions in behaviour that challenges and enhanced occupational engagement. All studies were completed when there was less clarity around language used to describe sensory interventions and before publication of the ASI ${ }^{\circledR}$ Fidelity Measure (Parham et al., 2011), but some are described in sufficient detail to demonstrate meeting many of the criteria for this intervention. Limitations of all these studies include interventions that may 
not reflect current practice, small sample sizes, and large variability of dose and length of treatment - thus limiting opportunities for systematic review. Only Clarke (1978) and Soper and Thorley (1996) used separate control groups, with the majority of other studies using single-case experimental designs. Some behavioural studies have also successfully used sensory strategies, such as vibration and deep pressure, to reduce behaviours that challenge, leading McGill and Breen (2020) to conclude sensory techniques can have a useful role in multi-element behavioural intervention for those with intellectual disabilities.

Growing numbers of intervention studies have been carried out in mental health (e.g. Machingura, 2018), though the majority of limitations described for the intellectual disability literature are similarly present. Additionally, there is often lack of clarity as to whether treatment described is more aligned to Ayres's theories or theories around sensory stimulation. Findings include reductions in seclusion, restraint and admissions to in-patient units and increased occupational performance. Findings are supported by positive service user reports of sensory interventions (e.g. Machingura et al., 2021).

More methodologically strong research has been carried out with children and to a lesser extent adults with autism. This includes a small number of randomised controlled trials and systematic reviews (e.g. Schaaf et al., 2018) of ASI ${ }^{\circledR}$. Findings indicated benefits of treatment, including higher achievement of functional goals and reduced assistance needed during daily activities, compared to usual care. Some weaker evidence has also emerged for use of specific sensory-based interventions. For example, improvements in classroom task performance when using a sensory activity schedule (Mills et al., 2021).

In spite of clear limitations, the overall picture of positive outcomes within the literature suggests some efficacy of sensory approaches with those with intellectual disabilities, autism and/ or mental health conditions and indicates further research is worthwhile. This Delphi study aimed to gather experienced occupational therapists' views regarding important elements of practice when using sensory integration-informed approaches with adults with intellectual disabilities admitted to ATUs.

\section{Method}

\section{Ethical approval}

Ethical approval was received from The University of Brighton School of Health Sciences Research Ethics and Governance Committee.

\section{Study design}

The Delphi approach was used, as it could capture views of therapists spread over a large geographical area (UK and Ireland) and has been recommended for use in health care when there is a dearth of empirical evidence (McCarthy et al., 2015). In Delphi studies, participants respond to a number of rounds of surveys and can change their views in later rounds after considering others' responses. A modified Delphi was chosen, in which predetermined information was provided for ranking in round one, along with a decision to limit the Delphi to three rounds. Items included in the Round One survey were identified from the sensory integration literature. As there was little published material regarding adults with intellectual disability, this included related areas such as children and young people, autism and mental health. The survey required participants to rate the importance of assessments, interventions, outcome measures, facilities, equipment and training when using sensory integration-informed approaches with adults with intellectual disabilities. Multiple pilot versions of the Round One survey were tested with four sensoryintegration trained, intellectual disability occupational therapists and adapted in light of feedback. For example, wording was amended to improve clarity, and the number of questions was reduced. Surveys were hosted on an online survey website.

\section{Participants}

A purposive, volunteer sample was used. This sought to recruit senior occupational therapists and above, with experience working, supervising or managing others on an ATU within the past five years. Written consent was not required. Participants were informed that completion of the surveys would be taken as consent for information they provided to be used in the research.

\section{Data collection and analysis}

Quantitative data collection used a six-point Likert-style scale, based on McCarthy et al. (2015), with 1 labelled "not at all important" and 6 "extremely important." An additional option of "never used, can't comment" was included. Qualitative data was collected via comments boxes. Participants were also requested to add additional items for rating. Following each round, participants received summarised data and reflected on the ratings and comments provided before completing the next round.

Items met consensus for importance if $80 \%$ of panellists rated them 4-6 on the Likert-style scale. No clear guidance exists regarding appropriate levels of consensus within Delphi studies. A high level of consensus was felt to be required owing to the homogeneous panel and predicted small panel size, which meant disagreement of a minor number of participants would constitute a significant percentage.

Quantitative data were analysed using descriptive statistics, whilst inductive thematic analysis (Braun and Clarke, 2006) was used to analyse the qualitative data. This was achieved by printing out collated comments and physically cutting up, arranging and re-arranging the text. Data was reviewed multiple times, coded, then developed into themes that were repeatedly reviewed, defined and refined. The second and third authors and two participants reviewed the data analysis; and final themes reflected feedback from these reviews.

\section{Findings}

A total of 13 senior occupational therapists, with recent experience working on an ATU and post-registration training in sensory integration-informed approaches, were recruited. All had been qualified five years or more, with a mean of 14 years working in intellectual disabilities. Six had a postgraduate qualification in sensory integration; and all had completed the first module of a sensory integration masters-level qualification. The panel therefore had demonstrable levels of expertise, of similar and in some cases higher levels to those in other Allied 
Health Profession Delphi studies (e.g. Timmer et al., 2015). A total of 13 participants completed Round One; 12 completed Round Two; and 10 completed Round Three. In light of the small population, this was a positive response and included the 10-15 participants described as effective for homogeneous groups by Ziglio (1996).

Participants strongly agreed on many elements of occupational therapy they felt were important, with many items reaching $100 \%$ consensus. See Table 1 for items rated important. No item received agreement by participants that it was "unimportant." Participants disagreed about the level of importance of a substantial number of items (46\%). This was particularly the case with assessments and outcome measures.

Participants also provided a large number of in-depth comments, many of which demonstrated aspects of their clinical reasoning. Two themes were identified from analysis of the comments, complexity, illuminating the complexity of working in this particular clinical environment, along with participants' complex clinical reasoning in response to this; and Pragmatism vs Ideal World, illustrating how participants' clinical decision-making is influenced by external factors, with a tension between current and desired practice.

\section{Theme 1: complexity}

This theme consisted of two sub-themes: Complexity of Challenge, highlighting key challenges to practice related to the complexity of people with intellectual disabilities, the ATU environment, available tools and multi-disciplinary working; Complexity of Solution demonstrating how participants overcome these difficulties via complex clinical reasoning, which included creativity and an individualised approach to each person.

\section{Complexity of challenge}

Challenges to practice included complexity of service users, where individuals have multiple diagnoses, complex health needs and a wide variety of cognitive, physical, communication and functional abilities:

Very few of my caseload would have the language ability to understand and answer the questions in the Adult/Adolescent profile.

It also included the complexity of providing sensory integration-informed interventions in an environment designed for individuals with complex needs:

I feel that some of my service users may benefit from Ayres SI however there is lots of red-tape around this in my clinical area - space, resources, infection control, risk assessment, ligature risk etc.

Many restrictions regarding available standardised tools were highlighted. This was particularly true of assessments, but also included outcome measures and manualised treatment approaches. Comments indicate tools have challenges for use with this population owing to flaws in the tools themselves or being originally designed for use with children or adults without intellectual disabilities:

I find some of the indicators are difficult for carers to score.

Participants' comments revealed the extent to which their work is embedded within the work of the multi-disciplinary team. Although positive in providing an integrated service to individuals, this created challenges in terms of setting goals for their therapeutic work and measuring its outcomes:

I find it hard to set occupational goals to measure outcomes as my work is so intertwined with the work of other MDT members.

Reflecting this sub-theme in the quantitative findings - a relatively small number of the available assessments (37.5\%) and outcome measures (29\%) were rated important, suggesting few of these were felt to be suitable in this setting.

A challenge also appeared to exist around gaining views of service users regarding their care. Although the highest rated outcome measure was service user feedback/patient stories, and this was supported by comments such as, "I think service user experience is the most important outcome," half of the round three participants $(n=5)$ had not used Talking Mats ${ }^{\circledR}$, an evidence-based tool that enables people with communication difficulties to give their views and therefore were unable to comment on its importance.

\section{Complexity as solution}

Complex clinical reasoning was required to overcome these practice challenges. This involved an approach that differed for each individual and related to the person's ability and motivation to engage; the nature of their difficulties; and involvement of family members/carers.

As a result of the wide variety of cognitive and communication abilities of individuals, participants reported needing a selection of tools that would allow them to assess and intervene in a range of ways. Participants aimed to be personcentred and offer an individualised service when choosing assessment tools, setting goals and planning and carrying out interventions:

I work with clients with a range of abilities as well as through those who support them, I need to have a range of tools so I can pick which is most appropriate.

A high proportion of the listed interventions (68\%), facilities $(80 \%)$ and equipment $(79 \%)$ were rated important, suggesting having a choice from a wide variety of tools for therapy is important for person-centred practice.

Owing to the wide variety of service user presentations and complex systems around individuals, participants described working with a wide spectrum of potential partners to achieve goals. Not all service users were able to actively collaborate in interventions, therefore choice of partners and the nature of the work carried out with them varied depending on each person's needs and personal situation. Partnership with service users appeared to be aimed for wherever possible, but family members, paid carers and ward staff were also involved to inform and support the occupational therapists' work:

I measure the success or otherwise of the intervention used by setting goals with client/family/carers at the beginning, and evaluating the effectiveness of the 'treatment' (education/environmental changes etc.) throughout.

Participants also valued using items and techniques that could continue to be used following discharge:

A good range of small mobile items are essential to discover what might work well in community facilities.

Although the focus of the study was on sensory integrationinformed approaches, participant comments articulated a strong focus on occupation. The items rated important also 
Table 1 Items rated important

Assessments
- Gathering accurate history of recent and previous presentation
- Observations during everyday activity
- Clinically reasoned observations to response to sensory input
- Structured clinical observations
- Adult adolescent sensory profile
- Model of human occupation screening tool
- Antecedent behaviour consequence charts
- Sensory integration inventory revised
- Assessment of motor and process skills
- Behavioural functional assessment

Equipment

- Outside gym

- Equipment that can be used on ward, in individual spaces, in client homes

- Therapy/gym balls

- Tactile items (includes vibrating items)

- Weighted objects, e.g. balls/bean bags

- Ear defenders

- Props to support engagement in playful activity

- Weighted blanket/lap pad/shoulder pad

- Fidget boxes

- Range of sensory and activity equipment covering all the senses

- Mats

- Bouncing equipment

- Outdoor equipment

- Weighted jacket

- Vehicles or opportunities for drives

- Crash pillow/pad

- Rubber strips or ropes for pulling

- Spandex/Lycra

- Swings/suspended equipment

- Pilates bands

- Exercise bike or pedal machine

- Visual targets

- Oral motor items

- Seating that provides additional sensory input

- Home maintenance equipment

- Olfactory items

- Bear hug vests

- Gardening tools

- Climbing equipment

- "Squeeze Vest"

- Gustatory items

- Wobble board

\section{Interventions}

- Adding to multidisciplinary formulation/functional analysis

- Education for the person/family/paid carers/unit staff

- Environmental recommendations

- Adapting activities of daily living

- Adapting environments

- Compensatory strategies

- Sensory diet

- Adapting roles and routines

- Use of calming/modulating strategies

- Development of activity risk assessments

- Consultation

- Direct 1:1 clinical intervention using eclectic sensory-based approaches

- Seating with additional sensory input

- Use of the natural environment

- Visual arousal monitoring tools

Outcome measures

- Service user feedback/patient stories

- Goal attainment scaling

- Model of human occupation screening tool

- Antecedent behaviour consequence charts

Facilities

- Quiet rooms

- Therapy room without fidelity to ASI

- Chill out space/calming/"womb-like" area

- Garden/outside space

- Opportunities for walking/running

- Multi-gym

- Kitchen

- Sports hall

- Garden space that offers adult type sensory experiences

- Trampoline

- Outside swing

- Outside gym

Training

- Sensory Integration Module 1*

- Sensory Integration Modules $2 \& 3^{*}$

- Working with ASD and is it sensory is it behaviour

- The alert programme for self-regulation

- *Part of a UK postgraduate sensory integration practitioner pathway that has since been updated 
highlight this focus on occupation. Occupation-based assessments such as observations during everyday activity, the model of human occupation screening tool and assessment of motor and process skills were included. Environmental recommendations; adapting activities, environments, roles and routines; development of activity risk assessments are all core occupational therapy interventions; and many of the facilities and equipment items are linked to obvious occupational forms (e.g. cooking, exercising, gardening, playing, home maintenance). Use of sensory integration-informed approaches appeared integrated with core occupational therapy practice and were intertwined to develop a comprehensive formulation of the person's needs:

I think it is really important to understand the impact of the SI problem on occupational function.

Creativity was evident in many participant comments when describing the complexity of their work, e.g. in using a bespoke approach, using space and equipment flexibly and integrating occupational and sensory approaches. Participants also directly described needing to be creative to overcome the challenges inherent in this setting:

I think many of us in this clinical area are used to "making do" with the facilities we have and using them creatively.

\section{Theme 2: pragmatism vs ideal world}

Related to the complexity of this clinical environment, this theme demonstrated tensions for participants when deciding how to practice. Many aspects of therapists' clinical reasoning were affected by awareness of restrictions on their practice. For example, many clinical decisions were influenced by the limited time available:

Often there is a need for consultation and education, there can be a need for short quick input.

Space and equipment were used creatively so therapy aims could be achieved with limited resources:

Multi-sensory room: we do not have one, but as we have lots of sensory equipment and everyday activities can be used/adapted [...] I do not feel we need it.

Participants demonstrated an understanding that funding for equipment, facilities, therapist time and training was limited and effective use needed to be made of this:

Not all organisations can fund all of the SI modules for all OTs - there needs to be individual therapists with these qualifications so that effective supervision can be offered.

However, in contrast to comments accepting practical restrictions on practice, participants expressed their desire to work in different ways if able to:

But it would be fantastic to have a proper clinical space that would allow more work.

\section{Discussion and implications}

This research elicited occupational therapists' views regarding important elements of practice when using sensory integrationinformed approaches with adults with intellectual disabilities on ATUs. An experienced panel was in strong agreement regarding many elements of practice they felt were important for use in this setting.
Participants rated a wide variety of items important. This need for a large range of intervention possibilities reflects several of issues described in the themes, including variety of individuals seen; differing abilities and preferences of individuals; and lack of tools suitable for this group and setting. Disagreement about the level of importance of items was particularly evident with assessments and outcome measures. This supports findings elsewhere in the literature, with mental health service users and staff agreeing a variety of tools are needed to successfully implement sensory interventions (Machingura, 2021) and a need for more suitable sensory assessments identified by McGill and Breen (2020). Challenges around gaining views of service users and using systematic tools to support this also corroborate previous study findings, where therapists used mainly informal techniques to gather feedback (Ball and Shanks, 2012).

The clinical reasoning captured in the Complexity of Solution sub-theme illuminates the response to the above challenges, demonstrating some of the complex reasoning and skills required to shape intervention. Occupational therapists working in ATUs appear to require a highly developed skillset. This includes a strong level of creativity to match the complexity of the individuals, their wider system and the ATU environment, and to overcome the lack of resources available to them. Consideration of the support ATU occupational therapists require to ensure they can apply their skills most effectively would be beneficial. For therapists, this may include post-registration training in relevant sensory approaches and access to appropriately skilled clinical supervision. At a unit level, adequate levels of staffing to help overcome the time restrictions described and access to a suitable range of equipment and treatment spaces. For the profession, development of assessments, outcome measures and evidencebased interventions suitable for use with adults with intellectual disabilities are needed.

Examining these results in light of language use when describing sensory approaches, participants' practice could be seen to fit with a number of the approaches described in the literature. Some techniques and tools rated important are associated with sensory-based interventions and various items from the ASI ${ }^{\circledR}$ Fidelity Measure (Parham et al., 2011) reached consensus. The fact that participants rated $1: 1$ direct clinical intervention using eclectic sensory-based approaches as important, along with access to a therapy room without fidelity to $\mathrm{ASI}^{\circledR}$, suggests the current fidelity measure developed for use with children does not meet their needs. Findings also suggest when using sensory integration-informed approaches in this setting practice has a strong focus on occupation, with participants' sensory integration knowledge interwoven with their occupational expertise. This is in keeping with recommendations by the Royal College of Occupational Therapists (2021, p. 1) that occupational therapists "should maintain their occupational focus" and that sensory interventions "should be used as part of a wider approach to address a person's occupational needs."

This occupation-focussed sensory integration-informed approach fits well with current agendas to improve quality of care and enhance quality of life for people with intellectual disabilities. Those with intellectual disabilities, autism and sensory integration difficulties are at increased risk of 
developing behaviour that challenges (NICE, 2015) and therefore at risk of restrictive interventions. Occupational therapy assessment that includes consideration of an individual's sensory profile, preferences and needs fits with current NICE (2015) guidance, whilst intervention supporting participation in meaningful activity is a key element to enhance quality of life and may reduce behaviour that challenges (Ball and Fazil, 2013). Additionally, participants' clear focus on discharge supports the agenda to minimise the number of people within in-patient settings and links with the wider role of occupational therapists as discharge facilitators across other areas of the profession (Rogers et al., 2017).

\section{Conclusion}

This study contributes to the body of knowledge regarding how occupational therapists do and could work with people with intellectual disabilities and sensory integration difficulties. Although this study takes a UK and Ireland perspective, similar agendas regarding care and support for those with intellectual disabilities internationally mean findings are relevant more widely. Possible limitations include criticism of the Delphi approach for potential to compel consensus (Ziglio, 1996). To minimise this risk and reduce potential bias caused by using a modified Delphi, the survey design encouraged participants to add items and urged them to give their own views, even if disagreeing with others' ratings. Additionally, as participants were limited to rating importance of items they were familiar with; no item received consensus that it was "unimportant"; and some items that did not meet $80 \%$ consensus still reached high levels of agreement and/or received positive comments, it should be noted that items that did not reach consensus may still be important.

Participants strongly agreed on the importance of many elements of practice, with a substantial number of items reaching $100 \%$ consensus. The list of important items created can inform occupational therapists working in these settings and researchers developing intervention research.

Complementing the quantitative findings, the two themes have provided insight into practice in this setting, where work appears characterised by complexity and a relatively limited set of available clinical tools. Development of more appropriate assessments, outcome measures and evidence-based interventions is required. Training opportunities around sensory integration-informed approaches and evidence-based service-user evaluation tools are also needed. Ensuring adequate staffing levels may support occupational therapists to maximise what their role can potentially offer.

Further research around occupational therapy informed by sensory integration theory for those with intellectual disabilities would be beneficial to add to the theoretical understanding informing practice and to investigate the efficacy of this approach. Consideration of how to develop a concept of fidelity for $\mathrm{ASI}^{\circledR}$ to use with adults with intellectual disabilities may also warrant study. Finally, with a need to ensure people are discharged from ATUs as quickly as possible, exploring an increased occupational therapy role in discharge facilitation may support this agenda further.

\section{References}

Ayres, A.J. (1973), Sensory Integration and Learning Disorders, 8th ed. Western Psychological Services, Los Angeles, CA.

Ball, J. and Fazil, Q. (2013), "Does engagement in meaningful occupation reduce challenging behaviour in people with intellectual disabilities? A systematic review of the literature", Fournal of Intellectual Disabilities, Vol. 17 No. 1, pp. 64-77.

Ball, J. and Shanks, A. (2012), "Gaining feedback from people with learning disabilities", British Fournal of Occupational Therapy, Vol. 75 No. 10, pp. 471-477.

Bowen, B. and Kemp, S. (2014), "Replacing restraint: good practices in North America human service programs for persons with intellectual disabilities and autism", in Karmin, S.A. (Ed.), Human Rights Perspective on Reducing Restrictive Practices in Intellectual Disability and Autism.

Braun, V. and Clarke, V. (2006), "Using thematic analysis in psychology", Qualitative Research in Psychology, Vol. 3 No. 2, pp. 77-101.

Breslin, L., Guerra, N., Ganz, L. and Ervin, D. (2020), "Clinical utility of multisensory environments for people with intellectual and developmental disabilities: a scoping review", American fournal of Occupational Therapy, Vol. 74, p. 7401205060 , doi: 10.5014/ajot.2020.037267.

Brown, C., Karim, R. and Steuter, M. (2020), "Retrospective analysis of studies examining sensory processing preferences in people with a psychiatric condition", American fournal of Occupational Therapy, Vol. 74 No. 4, pp. 7404205130p1-7404205130p11.

Brown, A., Tse, T. and Fortune, T. (2018), "Defining sensory modulation: a review of the concept and a contemporary definition for application by occupational therapists", Scandinavian fournal of Occupational Therapy, Vol. 26 No. 7, doi: 10.1080/11038128.2018.1509370.

Clarke, F.A., Miller, L.R., Thomas, J.A., Kucherawy, D. and Azen, S.P. (1978), "A comparison of operant and sensory integrative methods on developmental parameters in profoundly retarded adults", American fournal of Occupational Therapy, Vol. 32 No. 2, pp. 86-92.

Gawlas, C. and Blaskowitz, M. (2020), “The impact of sensory diets on adults with intellectual and developmental disabilities (IDD)", American fournal of Occupational Therapy, Vol. 74 No. 4_Supplement_1, p. 1.

Gonthier, C., Longuépée, L. and Bouvard, M. (2016), "Sensory processing in low-functioning adults with autism spectrum disorder: distinct sensory profiles and their relationships with behavioral dysfunction", fournal of Autism and Developmental Disorders, Vol. 46 No. 9, pp. 3078-3089.

Green, D., Beaton, L., Moore, D., Warren, L., Wick, V., Sanford, J.E. and Santosh, P. (2003), "Clinical incidence of sensory integration difficulties in adults with learning disabilities and illustration of management", British fournal of Occupational Therapy, Vol. 66 No. 10, pp. 454-463.

Haines, D., Wright, J. and Comerasamy, H. (2018), "Occupational therapy empowering support workers to change how they support people with profound intellectual and multiple disabilities to engage in activity: empowering support workers to enable engagement", Fournal of Policy and Practice in Intellectual Disabilities, Vol. 15 No. 4, pp. 295-306, doi: 10.1111/jppi.12257. 
Hebert, K. (2015), “The association between impulsivity and sensory processing patterns in healthy adults", British fournal of Occupational Therapy, Vol. 78 No. 4, pp. 232-240.

Johnson, K.R., Blaskowitz, M. and Mahoney, W.J. (2019), "Occupational therapy practice with adults with intellectual disability: what more can we do?", The Open fournal of Occupational Therapy, Vol. 7 No. 2, p. 12.

Lakhan, R. and Ekúndayò, O.T. (2017), "Important research priorities in intellectual disability in India: a public health perspective", Fournal of Neurosciences in Rural Practice, Vol. 8 No. Suppl, p. 1.

McCarthy, A., Moore, A., Redhead, L., McLaughlin, P., Iorio, A. and Chowdary, P. (2015), "Development of haemophilic arthropathy of the ankle: results of a Delphi consensus survey on potential contributory factors", Haemophilia, Vol. 21 No. 1, pp. 116-123.

McGill, C. and Breen, C.J. (2020), “Can sensory integration have a role in multi-element behavioural intervention? An evaluation of factors associated with the management of challenging behaviour in community adult learning disability services", British fournal of Learning Disabilities, Vol. 48 No. 2, pp. 142-153.

Machingura, T., Shum, D., Molineux, M. and Lloyd, C. (2018), "Effectiveness of sensory modulation in treating sensory modulation disorders in adults with schizophrenia: a systematic literature review", International Fournal of Mental Health and Addiction, Vol. 16 No. 3, pp. 764-780.

Machingura, T., Lloyd, C., Murphy, K., Goulder, S., Shum, D. and Green, A.H. (2021), "Views about sensory modulation from people with schizophrenia and treating staff: a multisite qualitative study", British fournal of Occupational Therapy, p. 0308022620988470.

Mills, C., Chapparo, C. and Hinitt, J. (2021), "Impact of a sensory activity schedule intervention on cognitive strategy use in autistic students: a school-based pilot study", British Fournal of Occupational Therapy.

NHS Digital (2020), "Learning disability services monthly statistics", available at: https://digital.nhs.uk/data-and-infor mation/publications/statistical/learning-disability-servicesstatistics

NHS (2019), The NHS Long Term Plan, NHS.

NICE (2015), Challenging Behaviour and Learning Disabilities: prevention and Interventions for People with Learning Disabilities Whose Behaviour Challenges, National Institute for Health and Care Excellence.

Parham, L.D., Roley, S.S., May-Benson, T.A., Koomar, J., Brett-Green, B., Burke, J.P., Cohn, E.S., Mailloux, Z., Miller, L.J. and Schaaf, R.C. (2011), "Development of a fidelity measure for research on the effectiveness of the Ayres sensory integration ${ }^{\circledR}$ intervention", American fournal of Occupational Therapy, Vol. 65 No. 2, pp. 133-142.

Reisman, J. (1993), "Using a sensory integrative approach to treat self-injurious behavior in an adult with profound mental retardation", American Fournal of Occupational Therapy, Vol. 47 No. 5, pp. 403-411.

Reisman, J.E. and Hanschu, B. (1993), "Using the consultative model to introduce sensory integration services for adults with developmental disabilities", Occupational Therapy Practice, Vol. 4 No. 4, pp. 38-46.

Rogers, A.T., Bai, G., Lavin, R.A. and Anderson, G.F. (2017), "Higher hospital spending on occupational therapy is associated with lower readmission rates", Medical Care Research and Review, Vol. 74 No. 6, pp. 668-686.

Royal College of Occupational Therapists (2021), Informed View: Sensory Integration and Sensory Based Interventions, College of Occupational Therapists, London.

Schaaf, R.C., Dumont, R.L., Arbesman, M. and May-Benson, T.A. (2018), "Efficacy of occupational therapy using Ayres sensory integration ${ }^{\circledR}$ : a systematic review”, American fournal of Occupational Therapy, Vol. 72 No. 1, p. 7201190010 , doi: 10.5014/ajot.2018.028431.

Soper, G. and Thorley, C.R. (1996), "Effectiveness of an occupational therapy programme based on sensory integration theory for adults with severe learning disabilities", British fournal of Occupational Therapy, Vol. 59 No. 10, pp. 475-482.

Timmer, A.J., Unsworth, C.A. and Taylor, N.F. (2015), "Occupational therapy inpatient rehabilitation interventions with deconditioned older adults following an acute hospital admission: a Delphi study", Australian Occupational Therapy fournal, Vol. 62 No. 1, pp. 41-49.

Urwin, R. and Ballinger, C. (2005), "The effectiveness of sensory integration therapy to improve functional behaviour in adults with learning disabilities: five single-case experimental designs", British Fournal of Occupational Therapy, Vol. 68 No. 2, pp. 56-66.

Watling, R. and Hauer, S. (2015), "Effectiveness of Ayres sensory integration ${ }^{\circledR}$ and sensory-based interventions for people with autism spectrum disorder: a systematic review", American fournal of Occupational Therapy, Vol. 69 No. 5, p. $6905180030 \mathrm{p} 1$.

Ziglio, E. (1996), "The Delphi method and its contribution to decision-making", in: Adler, M. and Ziglio, E. (Eds), Gazing into the Oracle: The Delphi Method and Its Application to Social Policy and Public Health, Jessica Kingsley Publishers, London.

\section{Corresponding author}

Helen Justice can be contacted at: helen.justice@nhs.net 